\title{
Performance and the Optimal Cut-Off Value of First- Trimester Fasting Plasma Glucose To Identify Gestational Diabetes Mellitus: A Retrospective Study From Southern China
}

\author{
Jia-Ning Tong \\ Southern Medical University \\ Lin-Lin Wu \\ Southern Medical University \\ Yi-Xuan Chen \\ Southern Medical University \\ Xiao-Nian Guan \\ Southern Medical University \\ Kan Liu \\ Southern Medical University \\ Ai-Qi Yin \\ Southern Medical University \\ Hua-Fan Zhang \\ Southern Medical University \\ Jian-Min Niu ( $\square$ njianmin@163.com ) \\ Southern Medical University
}

\section{Research Article}

Keywords: First-trimester FPG, GDM, Metabolic diseases in pregnancy, Adverse pregnancy outcomes

Posted Date: November 23rd, 2021

DOI: https://doi.org/10.21203/rs.3.rs-1067857/v1

License: (c) (1) This work is licensed under a Creative Commons Attribution 4.0 International License. Read Full License 


\section{Abstract \\ Purpose}

Previous studies have suggested that first-trimester fasting plasma glucose (FPG) is associated with gestational diabetes mellitus (GDM) and is a predictor of GDM. The aim of the present study was to explore whether first-trimester FPG levels can be used as a screening and diagnostic test for GDM in pregnant women.

\section{Methods}

This retrospective study included pregnant women who had their first-trimester FPG recorded at 9-13 weeks and underwent screening for GDM using the 2-hour $75 \mathrm{~g}$ oral glucose tolerance test (OGTT) between 24th and 28th gestational weeks. The cut-off values were calculated using a receiver operating characteristic (ROC) curve.

\section{Results}

The medical records of 28,030 pregnant women were analysed, and 4,669 $(16.66 \%)$ of them were diagnosed with GDM. The mean first-trimester FPG was $4.62 \pm 0.37 \mathrm{mmol} / \mathrm{L}$. The total trend in the optimal cut-off value of first-trimester FPG in pregnant women was $4.735 \mathrm{mmol} / \mathrm{L}$, with a sensitivity of $49 \%$, a specificity of $67.6 \%$ and AUC of 0.608 (95\% Cl: $0.598-0.617, p \otimes 0.001)$. Moreover, as the maternal age increased, the optimal cut-off values increased, respectively. The results suggest that first-trimester FPG can be considered a marker for identifying pregnant women with GDM.

\section{Conclusion}

The level of first-trimester FPG increased slightly with maternal age and, as maternal age increased, the optimal cut-off values increased, especially after age 30. The first-trimester FPG should be considered a screening marker when diagnosing GDM in pregnant women.

\section{Introduction}

Gestational diabetes mellitus (GDM) is one of the most common medical diseases related to pregnancy. It was previously defined as "hyperglycaemia first detected during pregnancy" ${ }^{1}$. According to the WHO, approximately $16 \%$ of pregnant women worldwide are affected by $\mathrm{GDM}^{2}$. GDM usually manifests in the second half of pregnancy and is caused by extreme physiological insulin resistance. Early diagnosis and treatment of GDM is extremely important because GDM can lead to several severe maternal-foetal complications, such as neonatal hypoglycaemia, birth injuries, macrosomia, shoulder dystocia, 
respiratory distress syndrome, childhood obesity and perinatal mortality ${ }^{3}$. Despite the worldwide prevalence and severity of GDM, a universally accepted screening test is lacking. Screening tests and diagnostic criteria vary widely among clinicians and across geographic areas ${ }^{1}$. Thus, the optimal method to screen for GDM in the first trimester remains unclear.

The ACOG and ADA recommend that all pregnant women, regardless of risk factors, should be screened for GDM by OGTT at 24 to 28 weeks of gestation 4,5 . In 2010, IADPSG also recommended a 75-gram OGTT test at 24-28 weeks of gestation for the diagnosis of GDM in all pregnant women with no apparent history of diabetes ${ }^{6}$. However, some studies have pointed out that OGTTs cannot be widely implemented because of the complexity of the test, the need for a prior appointment, the long waiting time and the low cost-effectiveness ${ }^{7}$. Doctors are thus attempting to find a more acceptable alternative strategy for the diagnosis of GDM to reduce the number of pregnant women who need to undergo an OGTT.

In this regard, FPG has been reported to have good efficacy as a screening test for GDM, especially at low thresholds, which has a strong influence on the exclusion of GDM in women. High-precision FPG can reduce the burden on the laboratory and save resources because it may be very difficult to carry out 75gram OGTTs with a large population and limited resources ${ }^{8}$. Previous studies have shown that FPG can be used to predict the risk of GDM in the third trimester, but there are significant differences among geographical regions of the world ${ }^{7,8}$. Compared with the use of OGTTs, FPG is easy to manage, welltolerated, reliable and has good repeatability; it also changes minimally throughout the entire pregnancy ${ }^{9}$. However, the usefulness of FPG in predicting GDM is not widely recognized because of the different diagnostic criteria, the choice of gestational age and differences related to race. There are no recognized diagnostic criteria for FPG in pregnant women ${ }^{9,10}$. First-trimester FPG for screening GDM lacks related research with large samples in southern China, where the prevalence of GDM is different from that in northern China because of in cooking habits, flavour styles and so on ${ }^{11,12}$. Southerners like sweets and are fed rice, whereas northerners prefer salty food. Therefore, it is necessary to establish some evidence for the use of first-trimester FPG and to delineate its optimal cut-off value for diagnosing GDM in southern China. In our study, we attempted to use the IADPSG standard ${ }^{6}$ to assess the sensitivity and specificity of FPG in the diagnosis of GDM to avoid the implementation of a large number of OGTT tests in southern China.

\section{Methods}

\section{Subjects}

This retrospective study included pregnant women who delivered between 1 June 2017 and 31 December 2019 at Shenzhen Maternal and Childcare Hospital in Shenzhen, southern China. Pregnant women younger than 18 years old, multiple pregnancies, pregnancies conceived by assisted reproductive technology, first-trimester FPG $>7.00 \mathrm{mmol} / \mathrm{L}$ and incomplete information, such as FPG in the firsttrimester (FPG value between $9-13^{+6}$ weeks of gestation) ,0-, 1- or 2-hour plasma glucose in a 2-hour $75 \mathrm{~g}$ 
OGTT between 24-28 weeks' gestation, were excluded. Finally, only 28,030 pregnant women were included in our study (Figure 1). The study was reviewed and approved by the Ethical Review Boards of Shenzhen Maternal and Childcare Hospital (Approval number: Shenzhen Maternal and Child Ethics Review No. 23; Approval date: 2017-04-07).

\section{Data Collection and Definitions}

Demographic information and medical data, such as maternal age, pregestational body mass index (BMI), first-trimester FPG (collected the sample before 10 am every day), and 0-, 1- or 2-hour plasma glucose in a 2-hour $75 \mathrm{~g}$ OGTT between 24-28 weeks' gestation, were collected. The first-trimester FPG and OGTT results were measured using the enzyme electrode method (DXC800, Beckman). First-trimester FPG was measured using venous blood samples after at least $8 \mathrm{~h}$ at the first visit and divided into seven groups according to the HAPO study $(₫ 4.2 \mathrm{mmol} / \mathrm{L}, 4.2-4.4 \mathrm{mmol} / \mathrm{L}, 4.5-4.7 \mathrm{mmol} / \mathrm{L}, 4.8-4.9 \mathrm{mmol} / \mathrm{L}, 5.0-$ $5.2 \mathrm{mmol} / \mathrm{L}, 5.3-5.5 \mathrm{mmol} / \mathrm{L}, \geq 5.6 \mathrm{mmol} / \mathrm{L})$. GDM is defined as $F P G, 1$ - or 2-hour plasma glucose of $\geq 5.1, \geq 10.0$, or $\geq 8.5 \mathrm{mmol} / \mathrm{L}$, respectively, in 2-hour $75 \mathrm{~g} \mathrm{OGTT}$ values between 24-28 weeks' gestation by IADPSG standard ${ }^{6}$. The study population was stratified according to GDM status: GDM (FPG, 1- or 2hour plasma glucose of $\geq 5.1, \geq 10.0$, or $\geq 8.5 \mathrm{mmol} / \mathrm{L}$, respectively) and non-GDM.

\section{Statistical Methods}

Data are presented as the mean \pm standard deviation (SD) for continuous variables and numbers (percentages) for categorical variables. Differences between the GDM and non-GDM groups were assessed by the Student's t-test-and the chi-squared test according to the nature of the variables. Cut-off values of the first-trimester FPG were determined using receiver operating characteristic (ROC) curves, and the area under the curve (AUC) and $95 \%$ confidence intervals (Cls), positive predictive value (PPV) and negative predictive value (NPV) were calculated. All statistical analyses were carried out using R software (version 4.0.3, https://www.r-project.org/). A two-tailed p-value $<0.05$ was considered statistically significant.

\section{Results}

\section{Baseline characteristics of pregnant women |between GDM and non-GDM}

Table 1 displays the different characteristics of the pregnant women between GDM and non-GDM. Among 28,030 pregnant women, 4,669 (16.66\%) were diagnosed with GDM. The mean maternal age was $31.01 \pm 4.12$ years, and the number of pregnant women with GDM was significantly higher than that of non-GDM women $(P<0.001)$. Among the GDM group, women aged 30-34 years occupied the largest number (42.13\%), and in the non-GDM group, women aged less than 30 years occupied the largest number (42.87\%). The mean first-trimester FPG level was $4.62 \pm 0.37 \mathrm{mmol} / \mathrm{L}$. The incidence of GDM increased with the first-trimester FPG level in the groups with first-trimester FPG levels $<4.2 \mathrm{mmol} / \mathrm{l}$, between $4.2 \mathrm{mmol} / \mathrm{L}$ and $4.4 \mathrm{mmol} / \mathrm{L}$, and between $4.5 \mathrm{mmol} / \mathrm{L}$ and $4.7 \mathrm{mmol} / \mathrm{L}$ was $6.47 \%, 17.95 \%$, and $25.68 \%$ respectively $(P<0.001)$. The incidence of GDM decreased in the groups with first-trimester FPG 
levels 4.8-4.9 mmol/l, 5.0-5.2 mmol/L, 5.3-5.5 mmol/L and $\geq 5.6 \mathrm{mmol} / \mathrm{L}$, which was $23.82 \%, 15.40 \%$, $6.83 \%$ and $3.86 \%$, respectively $(P<0.001)$. The results of the OGTT at $24-28$ gestational were also significantly different for pregnant women between GDM and non-GDM (all $\mathrm{P}<0.05$ ). According to the IADPSG diagnosis standard of GDM, the prevalence of GDM with $0 \mathrm{~h}, 1 \mathrm{~h}$ and $2 \mathrm{~h}$ of OGTT was $21.27 \%$, $38.23 \%$ and $49.39 \%$, respectively.

\section{Predictive Performance and the Optimal first trimester FPG Cut-Off Points}

Figure 2 showed the association between odds ratio (OR) of GDM and levels of first-trimester FPG ( $\mathrm{mmol} / \mathrm{L}$ ), as first-trimester FPG increased, the OR for identifying GDM increased among pregnant women, with a value of first-trimester FPG at $4.6 \mathrm{mmol} / \mathrm{L}$ which OR equal to 1 (Chi-Square $=665.79, \mathrm{p}<$ 0.001). The optimal cut-off value of maternal age-specific first-trimester FPG for identifying GDM were shown in Table 2. And Figure 3 showed first-trimester FPG for identifying GDM in pregnant women among different maternal age. The optimal cut-off value of first-trimester FPG to distinguish GDM in pregnant woman was $4.735 \mathrm{mmol} / \mathrm{L}$, with a sensitivity of $49 \%$ and a specificity of $67.6 \%$ and an AUC of 0.608 (95\% Cl: $0.598-0.617$ ), the optimal cut-off value of first-trimester FPG for pregnant women for identifying GDM were higher with maternal age $(Z$ test $=24.065, p<0.01)$.

\section{The incidence of GDM by different screening and diagnostic criteria}

Set first-trimester FPG greater than or equal to $4.7 \mathrm{mmol} / \mathrm{L}$ (first trimester FPG $>=4.7 \mathrm{mmol} / \mathrm{L}$ ) as a screening criterion. Figure 4 showed the incidence of GDM by different screening and diagnostic criteria, it was indicated that $51.68 \%$ of the pregnant women can be diagnostic as GDM with first-trimester FPG, which overlapped $21.27 \%, 38.23 \%$ and $49.39 \%$ in OGTT $0 \mathrm{~h}, 1 \mathrm{~h}$ OGTT and $2 \mathrm{~h}$ OGTT at $24 \sim 28$ gestational weeks, respectively.

\section{Discussion}

In 2008 , the HAPO study was conducted with 25,505 pregnant women in 15 centres from nine countries who underwent $75 \mathrm{~g}$ OGTT at 24 to 32 gestation weeks and showed that an elevated FPG lower than the diagnosis level of diabetes in pregnant women was associated with adverse pregnancy outcomes, including GDM, LGA and/or macrosomia, as well as caesarean delivery, and was strongly and consistently associated with birth weight gain and elevated C-peptide levels in cord blood ${ }^{13}$. Another study of HAPO further noted that higher FPG levels in the first trimester, which are currently considered to be nondiabetic, increased the risk of adverse pregnancy outcomes ${ }^{14}$. Our data presented here showed that the prevalence of GDM was $16.66 \%$ in southern China, which was similar to that of the $\mathrm{WHO}^{2}$. Interestingly, the results showed that the prevalence of GDM increased with the first-trimester FPG level increasing when it was lower than $4.7 \mathrm{mmol} / \mathrm{L}(\mathrm{P}<0.001)$. In particular, when the first-trimester FPG was between 4.2-4.4 mmol/L, the incidence of GDM sharply increased until the first-trimester FPG reached 4.5$4.7 \mathrm{mmol} / \mathrm{L}$, and the incidence of GDM went to the peak. This trend is consistent with the HAPO study, in which $\mathrm{FPG} \leq 4.4 \mathrm{mmol} / \mathrm{L}(80 \mathrm{mg} / \mathrm{dL})$ was associated with a lower risk of some adverse outcomes, to 
some degree ${ }^{6}$. We also found that the association between OR of GDM and levels of first-trimester FPG, as first-trimester FPG increased, the OR for identifying GDM increased among pregnant women, with a value of first-trimester FPG at $4.6 \mathrm{mmol} / \mathrm{L}$ which OR equal to $1(p<0.001)$. The optimal cut-off value of first-trimester FPG to distinguish GDM in pregnant woman was $4.735 \mathrm{mmol} / \mathrm{L}$, with a sensitivity of $49 \%$ and a specificity of $67.6 \%$ and an AUC of 0.608 , which was similar with some studies ${ }^{10,15}$.T he optimal cut-off value of first-trimester FPG for pregnant women for identifying GDM were higher with maternal age $(p<0.01)$, which trend was alike with Yang's ${ }^{1}$.Furthermore, if we set first-trimester FPG greater than or equal to $4.7 \mathrm{mmol} / \mathrm{L}$ as a screening criterion, it was indicated that $51.68 \%$ of the pregnant women can be diagnostic as GDM with first-trimester FPG.

Early diagnosis of GDM is essential to prevent related pregnancy complications. A study in 2009 reported that higher first-trimester FPG increased the risk of some complications and implied that women with high risks would not receive appropriate attention if they were not diagnosed during the first trimester ${ }^{14}$. Mills et al. found a physiological decrease in FPG during normal pregnancy, which indicated that the standard of FPG in pregnancy should be different from that in nonpregnant individuals ${ }^{16}$. Our last research revealed a strong relationship between adverse pregnancy outcomes and GDM. The research also reported that the univariate analysis showed that first-trimester FPG was strongly associated with risks of outcomes, including GDM, caesarean section, macrosomia, GHD, primary caesarean section and $L G A$ (all $O R>1$, all $P<0.05$ ). Furthermore, the risks of $G D M$, primary caesarean section and $L G A$ increased with first-trimester FPG, as it was found to be at 4.19-4.63 mmol/L. After adjustments for multiple factors, every stage of first-trimester FPG was associated with the risk of GDM. With increasing first-trimester $F P G$, the risks of GDM increased ${ }^{17}$.

As the age of onset of diabetes declines, the risk that some young women may develop undiagnosed type 2 diabetes during pregnancy is quite real. The importance of screening for and properly managing GDM cannot be underestimated because GDM can cause severe maternal and infant complications ${ }^{1,11}$. However, it is estimated that in some countries with limited health care resources, the lack of universal screening may miss up to $43 \%$ of people with $\mathrm{GDM}^{1}$. Although the OGTT is the gold standard diagnostic test for GDM, it is associated with several potential limitations, such as high costs and laboratory requirements ${ }^{8}$. Therefore, screening all pregnancies with OGTTs can be difficult. Although FPG is not the gold standard for diagnosing GDM, measuring first-trimester FPG may be critical for screening for previously undiagnosed pre-existing diabetes. FPG has been proposed as a screening test for GDM because it is less time consuming and more user friendly and reduces the medical costs associated with universal oral glucose tolerance testing $8,14,18$. Therefore, it is important to determine the diagnostic performance and optimal cut-off value of FPG for GDM screening, especially in the first trimester.

The optimal sensitive and specific cut-off value for FPG is still controversial ${ }^{10}$. The IADPSG and ADA have different views on the cut-off value at which the diagnosis is made. The IADPSG uses an FPG of $5.10 \mathrm{mmol} / \mathrm{l}$ during the first prenatal visit and throughout pregnancy as the diagnostic criterion for GDM, whereas the ADA recommends that first-trimester FPG can be used only to determine overt diabetes ( 7.00 
$\mathrm{mmol} / \mathrm{L}$ ) and that the OGTT be used for GDM screening and diagnosis at 24-28 gestational weeks $\mathrm{s}^{5,6}$. A study of 6,520 pregnant women from India showed that a cut-off value of FPG of $76 \mathrm{mg} / \mathrm{dL}(4.2 \mathrm{mmol} / \mathrm{L})$ had highly sensitive and negative predictive value (NPV) for the diagnosis of GDM, whereas a cut-off value of FPG of $92 \mathrm{mg} / \mathrm{dL}(5.1 \mathrm{mmol} / \mathrm{L})$ had high specificity and positive predictive value (PPV) for the diagnosis $^{14}$. In a meta-analysis, 8 of 29 studies used different cut-off values for diagnosing GDM, and two reported $91 \mathrm{mg} / \mathrm{dl}(5.05 \mathrm{mmol} / \mathrm{L})$ as the optimal cut-off value for the diagnosis ${ }^{10}$. Other studies reported cut-off values of $81 \mathrm{mg} / \mathrm{dl}(4.5 \mathrm{mmol} / \mathrm{L}), 83 \mathrm{mg} / \mathrm{dl}(4.6 \mathrm{mmol} / \mathrm{L}), 84.5 \mathrm{mg} / \mathrm{dl}(4.69 \mathrm{mmol} / \mathrm{L})$, $86.8 \mathrm{mmol} / \mathrm{dl}(4.82 \mathrm{mmol} / \mathrm{L})$ and $89 \mathrm{mg} / \mathrm{dl}(4.94 \mathrm{mmol} / \mathrm{L})$. In these studies, the sensitivity for most cutoff values was also in the range of $60-80 \% 9,10,15$, similar to ours. Our study also suggests that firsttrimester FPG may be an indicator of subsequent GDM. Riskin-Masiah,et al ${ }^{14}$ reported that mild hyperglycaemia in the first trimester may lead to adverse outcomes. Our research showed that the incidence of GDM increased with first-trimester FPG levels. If FPG levels in pregnancy are not well controlled, the opportunity to reduce the risk of adverse outcomes is likely to be missed ${ }^{11}$. A cohort study of 361 healthy pregnant women by Mills et al. indicated that maternal age was independently associated with $\mathrm{FPG}^{16}$, which our study also supported. Our results indicated that the optimal cut-off value of firsttrimester FPG for pregnant women for identifying GDM were higher with maternal age $(p<0.01)$. Impaired glucose tolerance usually occurs in the second trimester, only after that can treatment start. In fact, when these pregnancies were first assessed as having GDM, more than $20 \%$ of the foetuses showed signs of macrosomia, which may influence clinical decisions, such as the choice of delivery mode ${ }^{10}$. Screening for GDM in the first trimester can reduce the incidence of pregnancy complications, macrosomia, caesarean section and others ${ }^{10,14}$. Therefore, early detection of GDM may reduce the risks and enable strict guidance from the beginning of pregnancy. Some studies found that despite treatment, patients diagnosed with GDM at an early stage had poorer outcomes, suggesting that first-trimester FPG may be a marker of glucose tolerance before pregnancy and poor pregnancy outcomes ${ }^{3}$.

In addition, lifestyle interventions to prevent GDM have been shown to be most effective in the first trimester ${ }^{19}$. In a meta-analysis of more than 11,000 pregnant women, Song et al. concluded that lifestyle interventions can prevent GDM only if implemented before the 15 th week of gestation ${ }^{20}$. Research on late intervention has been generally disappointing, which means that it would be useful to have early pregnancy markers of GDM risk to determine who could benefit from early intervention ${ }^{19}$. Prevention studies have shown the positive prevention effects of diet on the incidence of GDM, LGA, SGA and preterm birth ${ }^{21,22}$. The two-step diagnosis of GDM in the second and third trimesters provides a narrow intervention window ${ }^{19}$. When receiving treatment, $20 \%$ of the foetuses showed signs of macrosomia and increased abdominal circumference, again emphasizing the need for early risk markers. In this regard, first-trimester FPG may be useful in selecting patients for early screening or LGA monitoring of GDM ${ }^{19}$. In addition, because metabolic changes during pregnancy result in a decrease in PG of approximately 2 $\mathrm{mg} / \mathrm{dl}$ between six and ten gestational weeks, a specific threshold of PG for the gestational week or a narrow interval for the evaluation of first-trimester FPG should be determined ${ }^{23}$. 
Therefore, we recommend that all pregnant women have FPG assessed at their first visit in the first trimester to determine the risk of diabetes and GDM.

\section{Limitations}

The limitations of this study have to be acknowledged: the retrospective nature of this study makes it possible that some of the test conditions may not be optimal, for example, in terms of fasting, which may have influenced our results. In the absence of some missing data, we were unable to analyse the impact of some potential risk factors on the relationship between FPG and GDM. We did not have multiple data points from a single panel to indicate the most appropriate week for screening. In addition, the accuracy and repeatability of the measurements need to be guaranteed. Laboratory quality control is absolutely essential and can ensure that pregnant women are truly fasting, which has often proven to be challenging because pregnant women typically do not fast prior to the first-trimester visit, so doctors have to request that they return the next day. Some of these women do not return, especially those from rural areas; in addition, samples must be taken before 10 am to prevent prolonged fasting. Fagan's nomogram showed that FPG is clinically useful ${ }^{10}$; however, the diagnostic accuracy of any screening test for GDM may depend on several other factors, such as ethnicity, the timing of testing and the presence or absence of risk factors for $\mathrm{GDM}^{10}$. The effect of these variables cannot be determined in our analysis. Based on our study, we recommend that all pregnant women undergo FPG testing in the first trimester, particularly at the first antenatal visit. First-trimester FPG greater than $4.7 \mathrm{mmol} / \mathrm{L}$ will be considered a warning level. These data come from southern China, and the results may apply only to pregnant women in southern China. Despite these limitations, this study provided valuable insights into the accuracy of first-trimester FPG in the screening and diagnosis of GDM in southern China.

\section{Conclusions}

Based on our research, we found that the level of first-trimester FPG increased slightly with maternal age and, as maternal age increased, the optimal cut-off values increased, especially after age 30 . We also suggest that the risks of GDM should be given increased attention and management as soon as the firsttrimester FPG value is more than $4.7 \mathrm{mmol} / \mathrm{L}$, even in the presence of a normal OGTT and regardless of the age at pregnancy. The first-trimester FPG should be considered a screening marker when diagnosing GDM in pregnant women. Further studies are needed to improve the accuracy of FPG in different ethnicities to strengthen the evidence.

\section{Abbreviations}

PG: Plasma Glucose

FPG: Fasting Plasma Glucose

GDM: Gestational Diabetes Mellitus 
ACOG: The American College of Obstetricians and Gynaecologists

WHO: World Health Organization

ADA: American Diabetes Association

IADPSG: International Association of Diabetes and Pregnancy Study Groups

OGTT: Oral Glucose Tolerance Test

\section{Declarations}

\section{Acknowledgments}

Not applicable

\section{Disclosure}

\section{Approval of the research protocol}

This research was approved by the Review Board for Human Investigation and the Ethics Committee of Shenzhen Maternal and Children Hospital.

\section{Informed Consent}

Informed consent was obtained from every patient, and the investigations were performed in accordance with the principles of the Declaration of Helsinki.

Approval date of Registry and the Registration No. of the study/trial

Approval date: 2017-04-07; Shenzhen Maternal and Child Ethics Review No. 23;

\section{Animal Studies}

N/A

\section{Funding}

The survey was supported by the National Natural Science Foundation of China(81830041,81771611),Shen Zhen Science and Technology Innovation Committee Special Funding

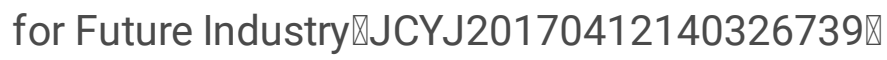

\section{Conflicts of interest}

None

\section{Author Contributions}


JM Niu and LL Wu were responsible for the study conception and design, they contributed equally to the manuscript. JN Tong did the statistical analysis and wrote the script. YX Chen and XN Guan collected the data. HF Zhang gave help to the data interpretation. K Liu and AQ Yin helped to revise part of the script. All authors contributed to the study design, including data collection, data interpretation and manuscript revision, and gave final approval of the version to be published.

\section{Availability of data and material}

All data generated or analysed during this study were included in this published article

\section{Code availability}

The codes used during and/or analysed during the current study are available from the corresponding authors (JM Niu) on reasonable request.

\section{Consent for publication}

Not applicable

\section{References}

1. Zhu WW, Yang HX, Wei YM, et al. Evaluation of the value of fasting plasma glucose in the first prenatal visit to diagnose gestational diabetes mellitus in china. Diabetes care. 2013;36(3):586-590.

2. Organization WHOJWH. Global report on diabetes. Geneva, Switzerland. 2016:1-88.

3. Bellamy L, Casas JP, Hingorani AD, Williams D. Type 2 diabetes mellitus after gestational diabetes: a systematic review and meta-analysis. Lancet (London, England). 2009;373(9677):1773-1779.

4. ACOG Practice Bulletin. Clinical management guidelines for obstetrician-gynecologists. Number 37, August 2002. (Replaces Practice Bulletin Number 32, November 2001). Thyroid disease in pregnancy. Obstetrics and gynecology. 2002;100(2):387-396.

5. 2. Classification and Diagnosis of Diabetes: Standards of Medical Care in Diabetes-2018. Diabetes care. 2018;41(Suppl 1):S13-s27.

6. Metzger BE, Gabbe SG, Persson B, et al. International association of diabetes and pregnancy study groups recommendations on the diagnosis and classification of hyperglycemia in pregnancy. Diabetes care. 2010;33(3):676-682.

7. Agarwal MM, Punnose J, Sukhija K, Sharma A, Choudhary NK. Gestational Diabetes Mellitus: Using the Fasting Plasma Glucose Level to Simplify the International Association of Diabetes and Pregnancy Study Groups Diagnostic Algorithm in an Adult South Asian Population. Canadian journal of diabetes. 2018;42(5):500-504.

8. Agarwal MM, Weigl B, Hod M. Gestational diabetes screening: the low-cost algorithm. International journal of gynaecology and obstetrics: the official organ of the International Federation of Gynaecology and Obstetrics. 2011;115 Suppl 1:S30-33. 
9. Benhalima K, Van Crombrugge P, Moyson C, et al. The Sensitivity and Specificity of the Glucose Challenge Test in a Universal Two-Step Screening Strategy for Gestational Diabetes Mellitus Using the 2013 World Health Organization Criteria. Diabetes care. 2018;41(7):e111-e112.

10. Li M, Lan JR, Liang JL, Xiong XL. Diagnostic accuracy of fasting plasma glucose as a screening test for gestational diabetes mellitus: a systematic review and meta-analysis. European review for medical and pharmacological sciences. 2020;24(21):11172-11186.

11. Yang W, Lu J, Weng J, et al. Prevalence of diabetes among men and women in China. The New England journal of medicine. 2010;362(12):1090-1101.

12. Sun T, Meng F, Zhao H, et al. Elevated First-Trimester Neutrophil Count Is Closely Associated With the Development of Maternal Gestational Diabetes Mellitus and Adverse Pregnancy Outcomes. Diabetes. 2020;69(7):1401-1410.

13. Metzger BE, Lowe LP, Dyer AR, et al. Hyperglycemia and adverse pregnancy outcomes. The New England journal of medicine. 2008;358(19):1991-2002.

14. Riskin-Mashiah S, Younes G, Damti A, Auslender R. First-trimester fasting hyperglycemia and adverse pregnancy outcomes. Diabetes care. 2009;32(9):1639-1643.

15. Li P, Lin S, Li L, Cui J, Zhou S, Fan JJPjoms. First-trimester fasting plasma glucose as a predictor of gestational diabetes mellitus and the association with adverse pregnancy outcomes. 2019;35(1):95.

16. Mills JL, Jovanovic L, Knopp R, et al. Physiological reduction in fasting plasma glucose concentration in the first trimester of normal pregnancy: the diabetes in early pregnancy study. Metabolism: clinical and experimental. 1998;47(9):1140-1144.

17. Tong JN, Wu LL, Chen YX, et al. Fasting plasma glucose in the first trimester is related to gestational diabetes mellitus and adverse pregnancy outcomes. Endocrine. 2021.

18. Wong T, Ross GP, Jalaludin BB, Flack JR. The clinical significance of overt diabetes in pregnancy. Diabetic medicine: a journal of the British Diabetic Association. 2013;30(4):468-474.

19. Cosson E, Carbillon L, Valensi P. High Fasting Plasma Glucose during Early Pregnancy: A Review about Early Gestational Diabetes Mellitus. Journal of diabetes research. 2017;2017:8921712.

20. Song C, Li J, Leng J, Ma RC, Yang X. Lifestyle intervention can reduce the risk of gestational diabetes: a meta-analysis of randomized controlled trials. Obesity reviews: an official journal of the International Association for the Study of Obesity. 2016;17(10):960-969.

21. Rayanagoudar G, Hashi AA, Zamora J, Khan KS, Hitman GA, Thangaratinam S. Quantification of the type 2 diabetes risk in women with gestational diabetes: a systematic review and meta-analysis of 95,750 women. Diabetologia. 2016;59(7):1403-1411.

22. Noctor E, Crowe C, Carmody LA, et al. Abnormal glucose tolerance post-gestational diabetes mellitus as defined by the International Association of Diabetes and Pregnancy Study Groups criteria. European journal of endocrinology. 2016;175(4):287-297.

23. Harrison CL, Lombard CB, East C, Boyle J, Teede HJ. Risk stratification in early pregnancy for women at increased risk of gestational diabetes. Diabetes research and clinical practice. 2015;107(1):61-68. 


\section{Tables}

Table 1 Clinical characteristics in pregnant women between GDM and Non-GDM 


\begin{tabular}{|c|c|c|c|c|}
\hline Features & Overall $(\mathrm{N}=\mathbf{2 8 0 3 0})$ & $\begin{array}{l}\text { Non- } \\
\text { GDM(N=23361) }\end{array}$ & GDM(N=4669) & $\mathbf{P}$ \\
\hline Maternal age, years, mean (SD) & $31.01 \pm 4.12$ & $30.71 \pm 4.03$ & $32.51 \pm 4.25$ & $<0.001$ \\
\hline$<30$, years, $\mathrm{n}(\%)$ & 11235 (40.08) & 10016 (42.87) & $1219(26.11)$ & \multirow[t]{4}{*}{$<0.001$} \\
\hline 30-34, years, n (\%) & 11155 (39.80) & 9188 (39.33) & 1967 (42.13) & \\
\hline 35-39, years, n (\%) & $4752(16.95)$ & 3567 (15.27) & 1185 (25.38) & \\
\hline$\geq 40$, years, $n(\%)$ & $888(3.17)$ & $590(2.53)$ & $298(6.38)$ & \\
\hline $\begin{array}{l}\text { Pregestational BMI, } \mathrm{kg} / \mathrm{m}^{2} \text {, } \\
\text { mean (SD) }\end{array}$ & $20.73 \pm 2.41$ & $20.62 \pm 2.37$ & $21.31 \pm 2.52$ & $<0.001$ \\
\hline$<24 \mathrm{~kg} / \mathrm{m}^{2}, \mathrm{n}(\%)$ & 25485 (90.92) & 21437 (91.76) & 4048 (86.70) & \multirow[t]{2}{*}{$<0.001$} \\
\hline$\geq 24 \mathrm{~kg} / \mathrm{m}^{2}, \mathrm{n}(\%)$ & $2545(9.08)$ & $1924(8.24)$ & $621(13.30)$ & \\
\hline $\begin{array}{l}\text { first } \\
\text { trimester FPG, mmol/L, mean } \\
\text { (SD) }\end{array}$ & $4.62 \pm 0.37$ & $4.59 \pm 0.36$ & $4.75 \pm 0.43$ & $<0.001$ \\
\hline$₫ 4.2 \mathrm{mmol} / \mathrm{L}, \mathrm{n}(\%)$ & $2812(10.03)$ & $2510(10.74)$ & $302(6.47)$ & \multirow[t]{7}{*}{$<0.001$} \\
\hline $4.2-4.4 \mathrm{mmol} / \mathrm{L}, \mathrm{n}(\%)$ & $6306(22.50)$ & $5468(23.41)$ & $838(17.95)$ & \\
\hline 4.5-4.7 mmol/L, n (\%) & $8802(31.40)$ & 7603 (32.55) & 1199 (25.68) & \\
\hline 4.8-4.9 mmol/L, n (\%) & $6110(21.80)$ & 4998 (21.39) & $1112(23.82)$ & \\
\hline 5.0-5.2 mmol/L, n (\%) & $2773(9.89)$ & $2054(8.79)$ & $719(15.40)$ & \\
\hline 5.3-5.5 mmol/L, n (\%) & $859(3.06)$ & $540(2.31)$ & $319(6.83)$ & \\
\hline$\geq 5.6 \mathrm{mmol} / \mathrm{L}, \mathrm{n}(\%)$ & $368(1.31)$ & $188(0.80)$ & $180(3.86)$ & \\
\hline \multicolumn{5}{|l|}{$\begin{array}{l}\text { OGTT at } 24 \sim 28 \text { gestational } \\
\text { weeks }\end{array}$} \\
\hline Oh OGTT, mmol/L, mean (SD) & $4.35 \pm 0.39$ & $4.29 \pm 0.32$ & $4.64 \pm 0.54$ & $<0.001$ \\
\hline $\mathrm{FPG}<5.1, \mathrm{mmol} / \mathrm{L}, \mathrm{n}(\%)$ & 27037 (96.46) & $23361(100.00)$ & 3676 (78.73) & \multirow[t]{2}{*}{$<0.001$} \\
\hline $\mathrm{FPG}>=5.1, \mathrm{mmol} / \mathrm{L}, \mathrm{n}(\%)$ & $993(3.54)$ & $0(0.00)$ & $993(21.27)$ & \\
\hline 1h OGTT, mmol/L, mean (SD) & $7.446 \pm 1.59$ & $7.11 \pm 1.31$ & $9.20 \pm 1.73$ & $<0.001$ \\
\hline 1h OGTT <10, mmol/L, n (\%) & 26245 (93.63) & $23361(100.00)$ & $2884(61.77)$ & \multirow[t]{2}{*}{$<0.001$} \\
\hline $\begin{array}{l}\text { 1h OGTT >= 10.0, } \mathrm{mmol} / \mathrm{L}, \mathrm{n} \\
(\%)\end{array}$ & $1785(6.37)$ & $0(0.00)$ & 1785 (38.23) & \\
\hline 2h OGTT, mmol/L, mean (SD) & $6.62 \pm 1.30$ & $6.32 \pm 1.00$ & $8.12 \pm 1.55$ & $<0.001$ \\
\hline $2 \mathrm{~h} \mathrm{OGTT}<8.5, \mathrm{mmol} / \mathrm{L}, \mathrm{n}(\%)$ & 25724 (91.77) & $23361(100.00)$ & $2363(50.61)$ & $<0.001$ \\
\hline
\end{tabular}


2h OGTT >= 8.5, $\mathrm{mmol} / \mathrm{L}, \mathrm{n}(\%) \quad 2306(8.23)$

$0(0.00)$

$2306(49.39)$

*The category of the first-trimester FPG was according to the HAPO study.

Table 2 Optimal cut-off first-trimester FPG for pregnant women with GDM among different maternal age

\begin{tabular}{|lllllll|}
\hline Population & $\begin{array}{l}\text { Optimal } \\
\text { Cutoff }\end{array}$ & AUC & specificity & sensitivity & PPV & NPV \\
\hline Total & 4.735 & $0.608(0.598 \sim 0.617)$ & 0.676 & 0.490 & 0.232 & 0.869 \\
\hline$<30$ & 4.775 & $0.597(0.579 \sim 0.615)$ & 0.752 & 0.408 & 0.167 & 0.913 \\
\hline $30-34$ & 4.735 & $0.601(0.587 \sim 0.615)$ & 0.656 & 0.505 & 0.239 & 0.861 \\
\hline $35-39$ & 4.915 & $0.587(0.567 \sim 0.606)$ & 0.801 & 0.346 & 0.366 & 0.787 \\
\hline 40 & 4.925 & $0.617(0.577 \sim 0.658)$ & 0.797 & 0.413 & 0.506 & 0.729 \\
\hline
\end{tabular}

Figures 


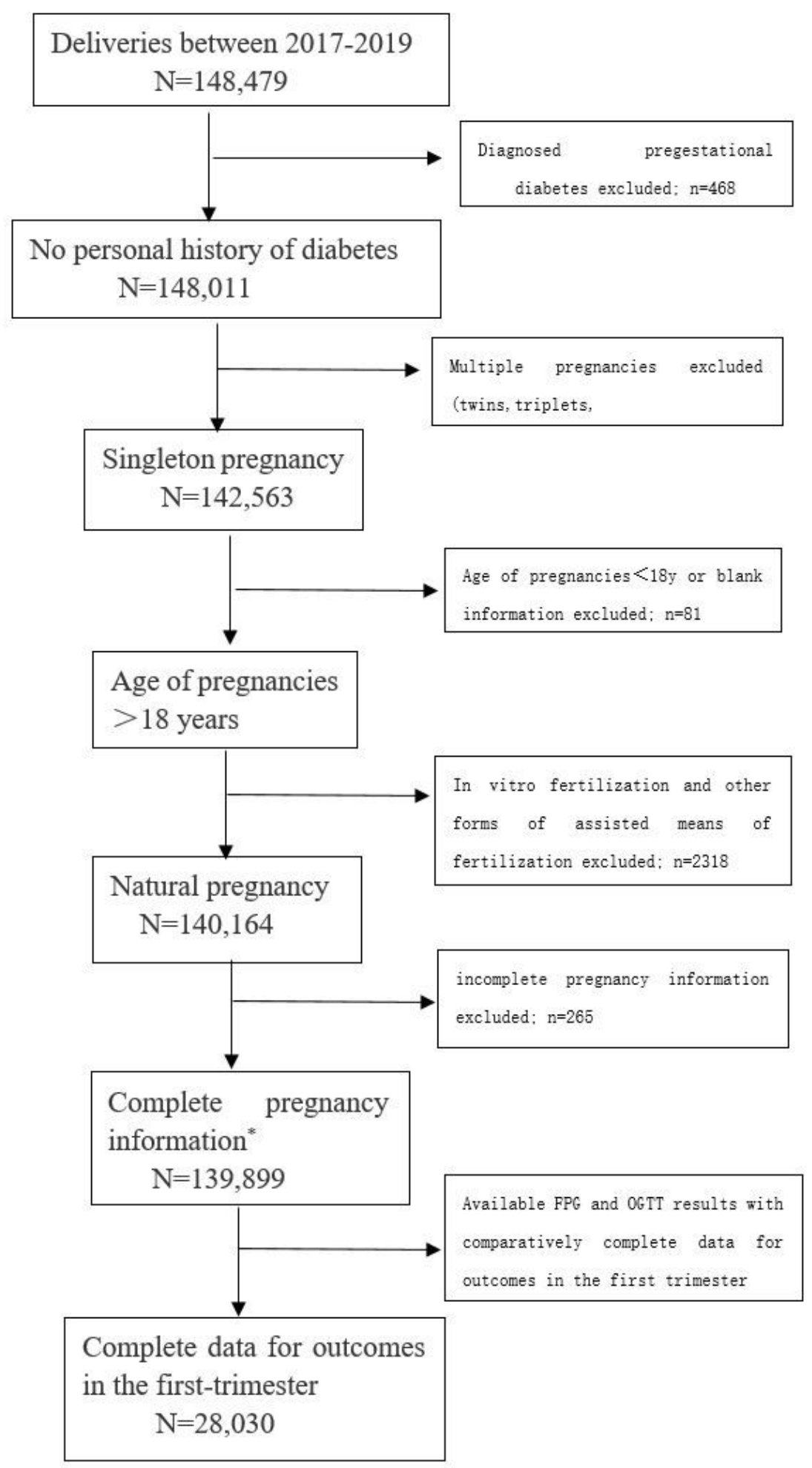

\section{Figure 1}

Flow chart of selecting process of the survey *: Complete data for outcomes refers to Gestational Diabetes Mellitus (GDM); Gestational hypertensive disorder (GHD); Macrosomia, Large for Gestational Age (LGA) and Low birth weight (LBW) and so on. 


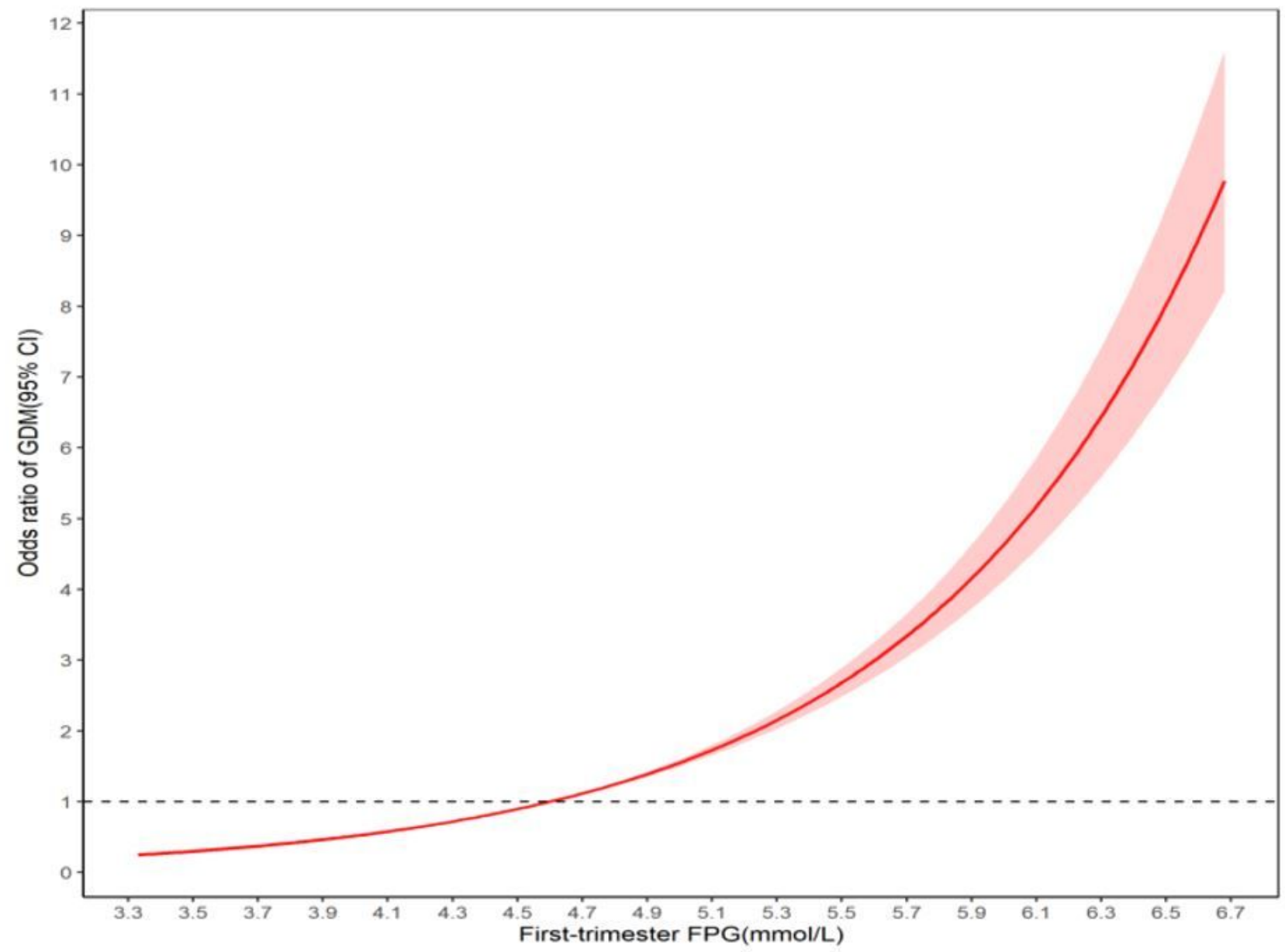

Figure 2

The association between odds ratio(OR) of GDM and levels of First-trimester FPG(mmol/L). The X-axis represented levels of first-trimester FPG and Y-axis represents OR of GDM by each level of first-trimester FPG. When First-trimester FPG is about 4.6, the OR equal to 1, the risk of GDM increased as levels of Firsttrimester FPG increased. 

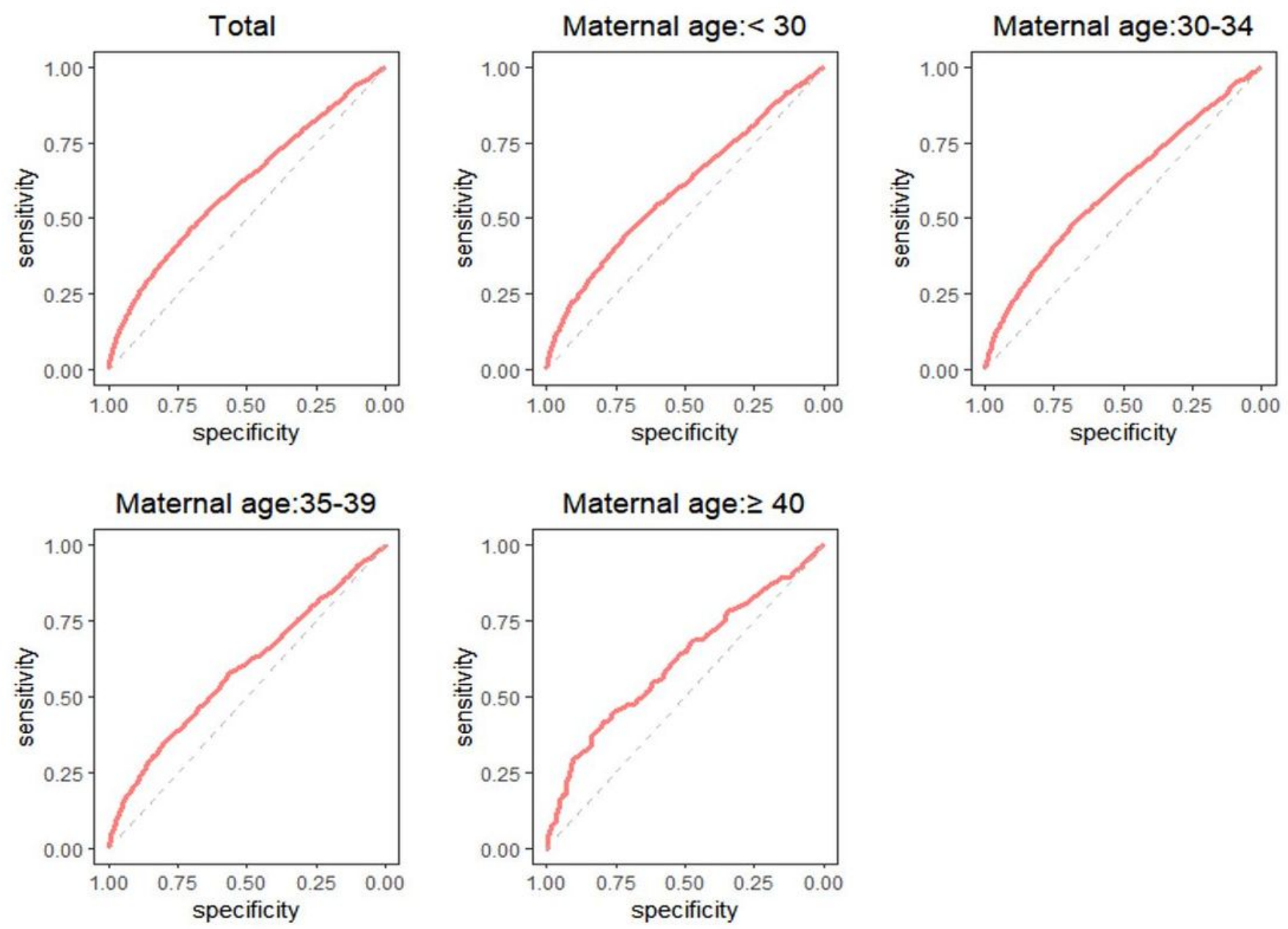

Figure 3

ROC curves of first-trimester FPG for identifying GDM in pregnant women among different maternal age. 


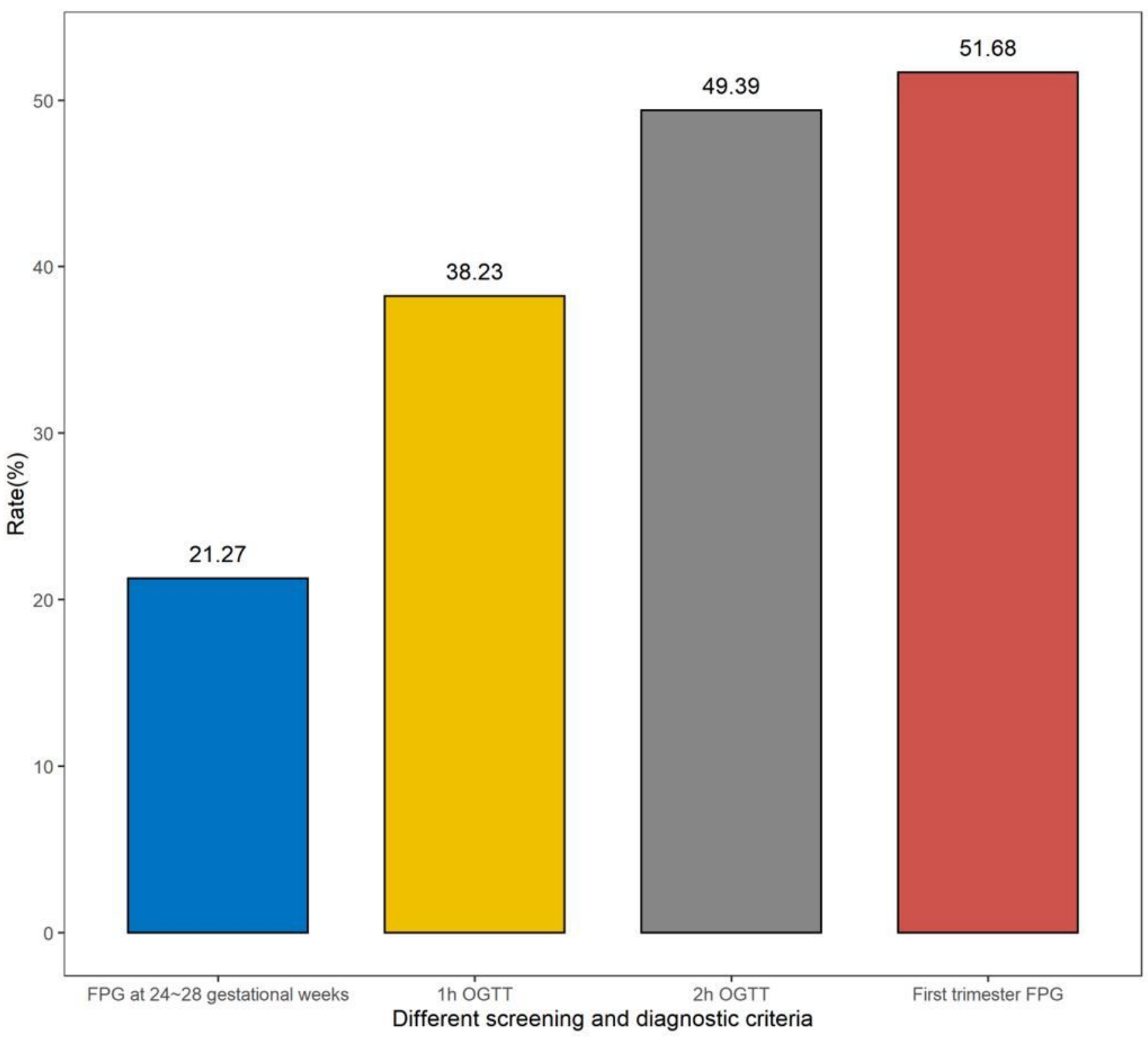

Figure 4

Incidence of GDM by different screening and diagnostic criteria. The X-axis showed different screening and diagnostic criteria-axis was rate of GDM by the x-axis screening and diagnostic criteria. *FPG at 2428 gestational weeks refers to the results of Oh OGTT. 